\title{
PERCEPÇÕES DE ESTUDANTES E DOCENTES SOBRE O REGIME DE TRATAMENTO EXCEPCIONAL PARA CURSOS DE ENGENHARIA
}

Cláudia Elianeda Matta - claudia.matta@unifei.edu.br

Universidade Federal de Itajubá (Unifei), Instituto de Sistemas Elétricos e de Energia (ISEE) Av. BPS, 1303, Bairro Pinheirinho

CEP: 37.500-903 - Itajubá-MG

Alessandra Rodrigues - alessandrarodrigues@unifei.edu.br

Universidade Federal de Itajubá (Unifei), Instituto de Física e Química (IFQ)

Av. BPS, 1303, Bairro Pinheirinho

CEP: 37.500-903 - Itajubá - MG

Rodrigo SilvaLima - rodlima@unifei.edu.br

Universidade Federal de Itajubá (Unifei), Instituto de Matemática e Computação (IMC)

Av. BPS, 1303, Bairro Pinheirinho

CEP: 37.500-903 - Itajubá-MG

Resumo: Durante o período de pandemia do novo coronavírus houve a substituição das aulas presenciais por aulas em meios digitais para os estudantes de todos os níveis de ensino. Para retomada das aulas, a Universidade de que trata este artigo elaborou um regime de tratamento excepcional, no qual os estudantes recebiam de cada docente um roteiro semanal de estudos com diversas estratégias de ensino, que poderiam ou não contemplar encontros virtuais síncronos. Após as primeiras semanas, foi elaborado um questionário on-line para estudantes e docentes sobre o regime implantado. Neste artigo, apresenta-se uma análise de parte dos dados de uma pesquisa descritiva e de cunho exploratório, com recorte nas percepções de estudantes e docentes de cursos de graduação. Visa-se investigar os pontos positivos e negativos apontados pelos estudantes acerca da educação mediada pelas tecnologias no contexto do ensino remoto emergencial e como os docentes utilizaram as tecnologias digitais de informação e comunicação para fazer a mediação pedagógica. Identificou-se que 50,9\% dos estudantes consideraram como ponto positivo dar seguimento aos estudos de forma remota e que a maior dificuldade enfrentada por eles foi o excesso de tarefas semanais. Os recursos digitais mais disponibilizados pelos docentes foram documentos em formato PDF $e$ apresentações de slides. Considera-se que o estudo é relevante por analisar as percepções e as práticas educativas com tecnologias digitais durante a pandemia, em um contexto brasileiro, e que pode contribuir para futuras discussões sobre as competências digitais para docentes em cursos de Engenharia.

Palavras-chave: Mediação pedagógica. Tecnologias digitais da informação e comunicação. Engenharia. Pandemia. 


\section{INTRODUÇÃO}

Em dezembro de 2019, na cidade de Wuhan, na China, houve um grande aumento de casos de pneumonia causado por um novo coronavírus (SARS-CoV-2). Essa doença, denominada pela sigla COVID-19, se espalhou rapidamente pelo mundo tornando-se uma pandemia. Por recomendação da Organização Mundial da Saúde (OMS), o distanciamento social passou a ser adotado como principal medida de contenção da disseminação da doença, tendo em vista a inexistência de vacinas ou de medicação específica para tratamento e cura. Essa medida teve impacto rápido e direto na vida social e econômica nos países de todos os continentes. De igual forma, gerou efeitos na educação em todos os níveis de ensino, uma vez que as escolas e universidades tiveram que suspender temporariamente suas atividades presenciais. É nesse contexto que, em 17 de março de 2020, o Ministério da Educação (MEC) brasileiro emitiu a Portaria $\mathrm{n}^{\mathbf{0}} 343$, que dispõe sobre a substituição das aulas presenciais por aulas em meios digitais, em decorrência da situação de pandemia do novo coronavírus (BRASIL, 2020). Depois dessa primeira, foram emitidas outras portarias, todas revogadas pela Portaria $n^{\circ} 544$, de 16 de junho de 2020, que estendeu até 31 de dezembro de 2020 a substituição das aulas presenciais por atividades remotas (incluindo-se aí as disciplinas laboratoriais cuja estrutura física e didática das instituições de ensino permita que sejam ofertadas remotamente).

$\mathrm{Na}$ universidade de que tratam as discussões deste artigo, as aulas foram suspensas por três semanas em 16 de março de 2020 e retomadas a partir de 04 de abril de 2020 por meio do que foi denominado Regime de Tratamento Excepcional (RTE), institucionalizado por seus conselhos deliberativos. O RTE possibilitou aos estudantes de graduação de cursos presenciais realizarem atividades acadêmicas domiciliares devido à impossibilidade de frequentar as aulas presenciais em decorrência do distanciamento social e das diretrizes sanitárias. De acordo com a Instituição, o RTE não é o mesmo que a oferta de Educação a Distância (EaD), embora utilize tecnologias de informação e comunicação para que as tarefas sejam realizadas. Nesse regime, os estudantes recebem de cada docente um roteiro semanal de estudos com diversas estratégias de ensino, que podem ou não contemplar encontros virtuais síncronos com os alunos. Vale lembrar que há uma diferença entre educação a distância e atividades remotas pela internet, já que na EaD há um modelo subjacente de educação que ampara as escolhas pedagógicas e organiza os processos de ensino e de aprendizagem, existem concepções teóricas e especificidades que sustentam essa modalidade. Já nas atividades remotas, as aulas presenciais são adaptadas provisoriamente para o meio digital (RODRIGUES, 2020a).

Neste artigo, analisamos as percepções de estudantes e docentes sobre as primeiras semanas do RTE, com o objetivo de investigar quais os pontos positivos e negativos da educação mediada pelas tecnologias digitais da informação e comunicação (TDIC) no contexto das disciplinas remotas e como foi realizada a mediação pedagógica pelos docentes a partir dos recursos utilizados e das ferramentas de comunicação. Os dados foram coletados por meio de um instrumento on-line disponibilizado no final do mês de abril de 2020, com questões direcionadas aos estudantes e aos docentes. Convém mencionar que a universidade na qual se realizou este estudo é, tradicionalmente, muito ligada às áreas exatas, com 34 cursos de graduação, dos quais 23 são de Engenharia.

Entendemos que analisar essas percepções e as práticas educativas com TDIC durante a pandemia em uma universidade no contexto brasileiro faz deste um estudo relevante por contribuir para as discussões sobre a formação de competências digitais para docentes em cursos de Engenharia. Sabemos que, mais que a tecnologia, o que facilita o processo de aprendizagem é a capacidade de mediação do professor. As TDIC, se bem utilizadas, facilitam 
a interaprendizagem, a colaboração, a reflexão, porém sem o planejamento adequado das atividades didáticas, podem não contribuir para que se atinja o resultado esperado.

\section{REFERENCIAL TEÓRICO}

A sociedade da informação, com um crescente uso das TDIC entre seus atores, tem impactado significativamente a vida das pessoas e modificado as formas de trabalho, de comunicação, de relacionamento, de aprendizagem e de pensamento (COLL; MONEREO, 2010). Essa sociedade também tem influenciado o cenário educacional, o que pode favorecer a utilização de recursos digitais nos processos de ensino e de aprendizagem, se houver uma formação dos professores para tal finalidade (MATTA; FURLANI; MORAES, 2020).

Assim, as TDIC provocam mudanças na educação, seja ela presencial ou a distância. Na modalidade presencial, as tecnologias desenraizam os conceitos de ensino e de aprendizagem limitados local e temporalmente tornando os espaços e tempos mais fluídos e permeáveis; e na $\mathrm{EaD}$ permitem o equilíbrio entre a aprendizagem individual e a colaborativa, na qual os estudantes de qualquer lugar podem aprender em rede (MORAN, 2013). A partir desse pressuposto, Azevedo et al. (2019) relatam a substituição de aulas expositivas por aulas mais interativas e participativas utilizando TDIC, com o intuito de favorecer uma aprendizagem mais significativa dos conteúdos e otimizar o tempo das aulas presenciais.

No início de 2020, os efeitos da pandemia nos levaram a refletir sobre as modalidades de educação presencial e a distância, nos fazendo perceber as oportunidades e os desafios impostos pelo isolamento social no que se refere às práticas educativas. As mudanças são de tal magnitude que precisaremos repensar os processos de ensino e de aprendizagem e os paradigmas convencionais de educação. É nesse contexto que Martins (2020) faz uma análise crítica sobre o comportamento preconceituoso em relação à $\mathrm{EaD}$, manifestado por boa parte das pessoas. Tal preconceito, fruto do senso comum e não da aplicação do conhecimento científico sobre educação, é observado, inclusive e infelizmente, entre docentes e gestores educacionais. Nessa mesma direção, Rodrigues (2020b) aponta que é necessário superar posturas ingênuas e salvacionistas quanto ao uso das TDIC na educação, mas também medrosas e demonizadas, assumindo sempre uma posição crítica, de reflexão e questionamento numa práxis contextualizada para uma educação transformadora e humanizadora.

Para superar este embate, é necessário ter clareza da importância da integração das tecnologias nos processos de ensinar e de aprender tendo como bússola a intencionalidade docente, a atitude de mediação pedagógica dos professores e a postura ativa dos estudantes. Com a inserção das TDIC no contexto escolar, o docente tem a oportunidade de realizar seu verdadeiro papel, o de mediador pedagógico (MASETTO, 2013). De acordo com este autor, faz parte da mediação pedagógica selecionar as técnicas que favoreçam o processo de aprendizagem de acordo com o que se pretende que os alunos aprendam em diferentes dimensões: intelectual, afetiva, atitudinal e de habilidades. Por mediação pedagógica entendemos a atitude, o comportamento do professor que se coloca como um facilitador da aprendizagem, com disposição para ser uma ponte entre o aprendiz e sua aprendizagem (MASETTO, 2013). Em outra definição, é a atitude e o comportamento do professor ao apresentar e tratar um conteúdo/tema que ajude o educando a compreender e lidar com as informações até produzir conhecimento e interferir em sua realidade (CRUZ, 2018). A postura docente de mediação exige também uma postura ativa e participante do aprendiz, uma postura de sujeito das ações que o levem a aprender e mudar seu comportamento (MASETTO, 2013). 


\section{ASPECTOS METODOLÓGICOS}

O presente artigo corresponde ao recorte de uma investigação com abordagem metodológica de natureza qualitativa, de cunho descritivo e exploratório (GIL, 2008). Os dados foram coletados por meio de dois questionários on-line disponibilizados no final do mês de abril de 2020, com questões direcionadas aos estudantes e aos docentes de uma universidade pública brasileira. Ressaltamos que a universidade na qual se realizou este estudo oferta 34 cursos de graduação, dos quais 23 são de Engenharia.

O número de estudantes de graduação que responderam o questionário foi de 3.246 , que corresponde ao $46 \%$ de um total de 7.110 alunos ativos na graduação. O número de professores que responderam o questionário foi de 365 , que corresponde a $77 \%$ de um total de 476 docentes ativos na Instituição. O primeiro questionário, destinado aos estudantes, continha 4 questões fechadas e uma questão aberta; o segundo possuía 8 questões fechadas e uma questão aberta e era direcionado aos docentes. Este artigo irá se ater às questões 3 e 4 dos questionários, que se voltavam, no caso dos estudantes, à indicação dos pontos positivos e das dificuldades relativas ao RTE; e no caso dos docentes, aos recursos utilizados para disponibilização de conteúdos e aos canais de comunicação com os estudantes durante o RTE.

\section{RESULTADOS E DISCUSSÃO}

Antes de iniciarmos a apresentação e discussão dos dados, cabe informar que para todas as questões analisadas neste texto, os respondentes podiam assinalar mais de uma opção. A porcentagem indicada é referente ao total de respondentes.

Quando perguntamos aos estudantes sobre os pontos positivos das atividades acadêmicas domiciliares, dar prosseguimento aos estudos de forma remota $(50,9 \%)$; criar uma rotina de estudos e, assim, se sentir produtivo durante a pandemia $(27,2 \%)$; e aprender a usar as tecnologias de maneira mais produtiva para facilitar os estudos $(15,5 \%)$ foram destacados como pontos fortes, conforme Figura 1.

Figura 1. Pontos positivos do RTE na percepção dos estudantes

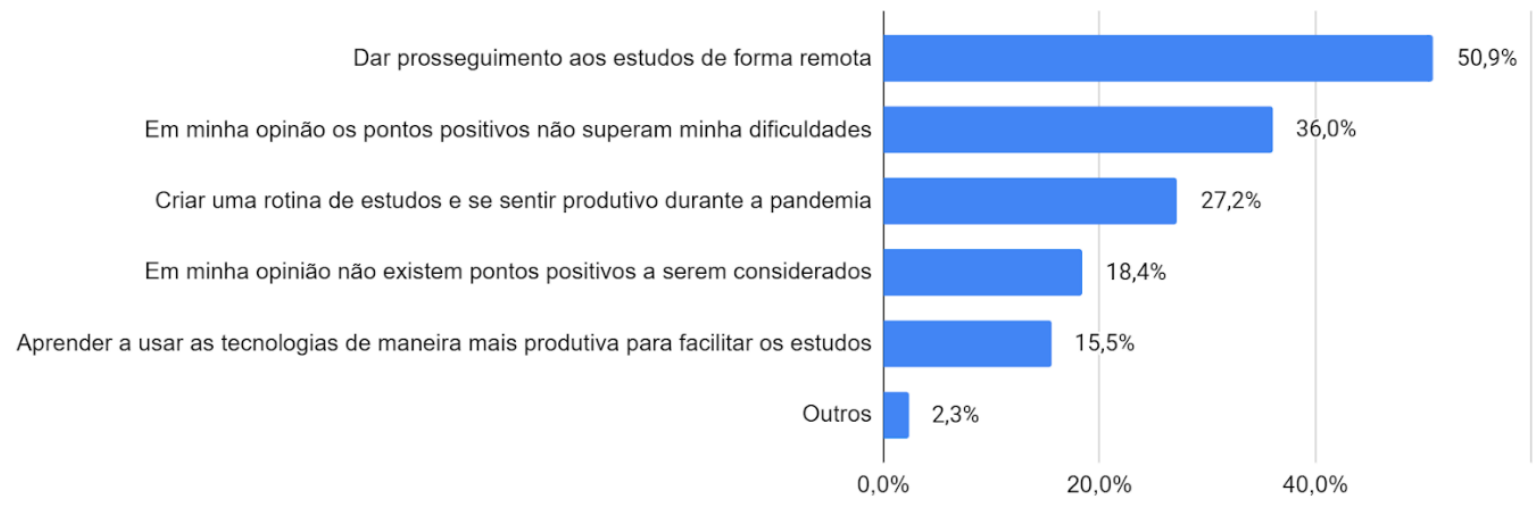

Fonte: Dados de pesquisa

Apesar de terem apontado aspectos positivos, $36 \%$ dos estudantes disseram que os pontos positivos não superam as dificuldades encontradas e 18,4\% afirmaram que não existem pontos positivos a serem considerados. De acordo com os referenciais nacionais dos cursos de Engenharia, a carga horária mínima é de 3.600 horas (BRASIL, 2007), o que constitui uma média 22 horas/aula semanais. Cursar essa carga horária de forma remota e sem 
necessariamente ter as condições emocionais e mesmo de infraestrutura (equipamentos e internet adequados) poderia causar esgotamento mental, falta de motivação ou evasão?

Quando perguntamos aos estudantes sobre as dificuldades encontradas em relação ao RTE, notamos que o excesso de tarefas semanais $(61,0 \%)$ foi a maior dificuldade apontada pelos discentes. Outra dificuldade foi a necessidade de conciliar tarefas domésticas enquanto estuda os materiais postados $(44,8 \%)$; ter que lidar com a ansiedade, depressão ou medo enquanto estuda (39,2\%); e não ter um ambiente saudável de estudo em casa $(33,5 \%)$ foram também outros pontos levantados pelos discente, conforme Figura 2.

Existe uma diversidade de alunos, com diferentes perfis, gostos, conhecimentos, culturas e idades; alguns ainda estão na fase de alfabetização e letramento digital, o que influencia a relação com os recursos digitais (SCHNEIDER; SILVA; BEHAR, 2013). No caso de sujeitos que têm mais experiência com os usos das TDIC, “[...] estes criam uma identidade virtual com maior facilidade, diferente daqueles que precisam percorrer um caminho mais longo devido a pouca experiência tecnológica" (SCHNEIDER; SILVA; BEHAR, 2013, p. 164). Embora os estudantes deste estudo possuam habilidades tecnológicas, eles optaram por um curso presencial. Sendo assim, será que eles possuem as competências necessárias para serem alunos virtuais? Esta foi uma das questões levantadas e uma dificuldade apresentada por $29,7 \%$ dos estudantes, que apontam a falta de familiaridade com conteúdos on-line como um entrave ao acompanhemento das disciplinas remotas, conforme Figura 2.

Figura 2. Dificuldades em relação ao RTE na percepção dos estudantes

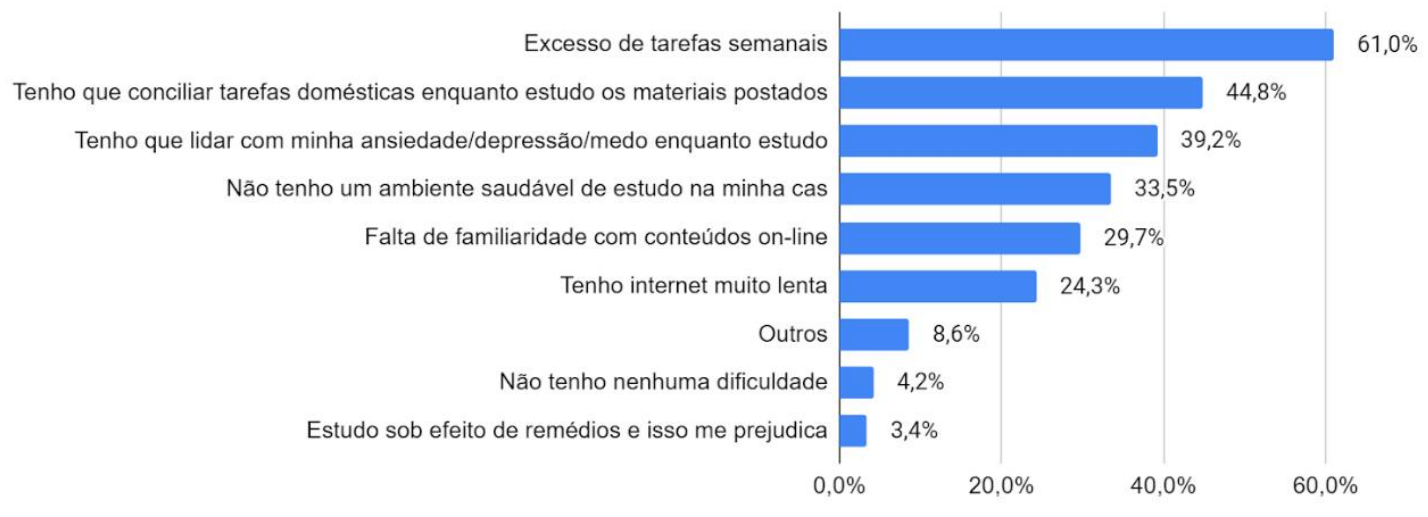

Fonte: Dados de pesquisa

Outra preocupação com a implantação do RTE foi referente aos estudantes que têm acesso limitado ou não têm acesso à internet por diferentes razões sociais e/ou econômicas. Dados apontam que $19 \%$ dos domicílios brasileiros possuem computadores de mesa, $27 \%$ computadores portáteis e 93\% celulares, já o acesso à internet é realidade em apenas $63 \%$ dos domicílios no Brasil (CETIC, 2019). Entre as dificuldades apontadas pelos alunos, 24,3\% relataram que têm uma internet lenta, conforme Figura 2.

Durante a pandemia, muitos docentes acostumados com aulas presenciais precisaram adaptar o conteúdo da disciplina para o meio digital. No RTE, coube ao docente definir quais, quando e onde os conteúdos da disciplina seriam disponibilizados. Sabemos que o grau das interações varia em função das mídias utilizadas: textos, áudios, vídeos, imagens, animações, entre outras, possibilitam diferentes graus de interação. Assim, no decorrer do processo de implantação do RTE perguntamos aos docentes qual foi o recurso utilizado para disponibilizar os conteúdos da disciplina. Eles responderam que os conteúdos foram disponibilizados em documentos PDF ou DOC (87,9\%), em apresentações de slides $(77,3 \%)$ e em vídeos $(49,9 \%)$, 
todos de autoria própria; 41,6\% disponibilizaram vídeos, 5,8\% apresentações de slides e 23,8\% documentos PDF ou DOC de terceiros; $10,7 \%$ disponibilizaram sistemas interativos de aprendizagem; $10,4 \%$ disponibilizaram simuladores on-line; 30,7\% utilizaram outros recursos e $0,8 \%$ não utilizaram recursos digitais.

Os conteúdos apresentados em arquivos PDF têm um formato seguro, multiplataforma, acessível e que possibilita a integridade do documento, porém não apresentam possibilidade de interação. Já os vídeos permitem o emprego de muitos formatos e linguagens, possuem estímulo auditivo e visual, podendo facilitar, assim, o aprendizado de estudantes com diversos estilos de aprendizagem. Além disso, os vídeos acentuam a percepção do conteúdo por meio das características próprias que esse recurso apresenta (MATTA; CLEMENTINO; MONTEVECHI, 2013). Em um estudo feito sobre a oferta da disciplina on-line de Cálculo em cursos de graduação presencial, $60 \%$ dos estudantes informaram que os vídeos gravados pelos docentes contribuíram para sua aprendizagem (MATTA et al., 2015). A linguagem audiovisual desenvolve múltiplas atitudes perceptivas, ao passo que a linguagem escrita desenvolve mais o rigor, a organização, a abstração e a análise lógica (MORAN, 2013).

Também chama a atenção nos dados o pequeno percentual de docentes que afirma ter utilizado sistemas interativos e simuladores (em torno de $10 \%$ para cada uma das possibilidades). Esse tipo de recurso favoreceria uma postura mais ativa e participativa dos estudantes e, em relação direta, uma atitude mais mediadora dos docentes, conforme sugere Masetto (2013). As respostas relativas aos recursos utilizados pelos docentes no RTE remetem a posturas docentes mais tradicionais e de replicação da aula expositiva presencial (arquivos de textos, 87,9\%; e slides, 77,3\%). Nessa direção, inferimos que os docentes, ao se depararem com o ensino remoto, num primeiro momento não buscaram formas mais colaborativas de promover a aprendizagem nem fizeram uso integrado e criativo das TDIC.

Nesse sentido, vale a reflexão sobre a importância da formação permanente de professores da educação superior, especialmente aqueles cuja formação inicial (e também em nível de pósgraduação) não se volta às especificidades da docência - como é o caso da maioria dos docentes deste estudo, cuja formação é em Engenharia e/ou outros cursos de bacharelado.

Há alguns caminhos que facilitam a aprendizagem, quais devemos escolher? Esta é uma questão que nos inquieta e que deve fazer parte da formação continuada e permanente dos docentes, já que um dos grandes desafios do educador é tornar a informação significativa para o aluno (MORAN, 2013). Para esse autor, aprendemos mais quando temos interesse, motivação clara, desenvolvemos hábitos que facilitam a aprendizagem e sentimos prazer no que estudamos e na forma como o fazemos.

Convém ressaltar que o papel do docente é promover a construção de significados, e não somente a transmitir conteúdos. É necessário ter disponibilidade para atender às solicitações dos estudantes, deixando claro em que momentos e como deverão fazer para obter ajuda (MORAN, 2013). Nas situações de ensino remoto, há uma vasta possibilidade de comunicação utilizando as TDIC, que podem ser síncronas ou assíncronas. Investigamos quais canais de comunicação foram utilizados pelos docentes para realizar a mediação pedagógica. Eles responderam que utilizaram o e-mail $(93,4 \%)$, o Google Meet $(67,7 \%)$, o fórum de dúvidas no Sistema Integrado de Gestão de Atividades Acadêmicas (SIGAA) (39,7\%), o fórum de dúvidas do Moodle $(4,9 \%)$, o Google Chat $(4,9 \%)$, o Telegram $(0,8 \%)$, outras formas de comunicação $(45,2 \%)$ e $0,2 \%$ não utilizaram nenhuma forma de comunicação.

Há evidências de que a combinação de comunicação síncrona e assíncrona apresenta níveis significativos de presença social na percepção do estudante (ROCKINSON-SZAPKIW et al., 2010). Como o construto de presença social está associado ao aumento da motivação, retenção, satisfação e aprendizado (GARRISON; ARBAUGH, 2007), é razoável concluir que a adoção 
da comunicação síncrona, como o Google Meet, tem o potencial de possibilitar uma educação on-line mais eficaz. Além disso, a mediação pedagógica deve ser dinâmica e promover a horizontalização da relação professor-aluno. Dessa forma, ferramentas de comunicação síncrona favorecem o diálogo e a interação.

Outra forma de comunicação apontada pelos docentes foi a criação de fóruns de dúvidas para interação com os estudantes. Os fóruns provêem uma forma de inteligência coletiva, na qual as dúvidas são compartilhadas pelos estudantes e respondidas pelos professores e podem auxiliar na aprendizagem dos demais participantes. Além disso, os próprios estudantes podem responder dúvidas dos colegas atuando, também eles, como mediadores e sujeitos ativos dos processos de ensinar e aprender. As tecnologias de comunicação flexível, como o fórum, possibilitam uma maior liberdade, já que cada um se expressa no momento mais oportuno (MORAN, 2013).

\section{CONSIDERAÇÕES FINAIS}

Finalizamos este artigo apontando os principais elementos advindos das percepções de discentes e docentes acerca do ensino remoto emergencial adotado em uma universidade pública brasileira na qual cerca de $70 \%$ dos cursos de graduação ofertados são da área de Engenharia. No que se refere às percepções dos discentes, cabe ressaltar, de um lado, o ponto positivo de dar continuidade aos estudos e sentir-se produtivo mesmo no contexto de pandemia e isolamento social. Por outro lado, a principal queixa dos discentes (excesso de tarefas semanais) pode estar associada tanto a questões contextuais decorrentes da pandemia (como instabilidade emocional e realização de tarefas domésticas) quanto com uma inadequação, por parte dos docentes, da relação tempo/conteúdos curriculares. O excesso de atividades parece indicar uma ênfase maior, dada pelos docentes, nos conteúdos a serem ministrados em detrimento das habilidades e competências a serem desenvolvidas ao longo da disciplina. Além disso, pode decorrer da pouca experiência de docentes e discentes com disciplinas remotas e/ou na modalidade $\mathrm{EaD}$ - que exigem atitudes mais autônomas dos discentes e posturas mais mediadoras dos docentes.

Relativamente aos recursos mais utilizados pelos docentes, salientamos o uso prioritário de arquivos de texto e práticas expositivas que replicam a aula presencial tradicional. Quanto aos canais de comunicação disponibilizados pelos docentes para atendimento aos alunos, ressaltamos o uso majoritário do e-mail (ferramenta assíncrona), mas também o uso do Google Meet (ferramenta síncrona) como os principais caminhos abertos ao diálogo. Em disciplinas remotas, a variedade de recursos bem como a amplitude das ferramentas de comunicação contribuem para a efetivação da mediação pedagógica e para a construção de um contexto de aprendizagem mais colaborativo e rico.

Para docentes e estudantes, estas mudanças não são fáceis, pois estão acostumados e se sentem seguros no ensino tradicional. Entretanto, a experiência emergencial com o ensino remoto pode contribuir para que os docentes reflitam mais diretamente sobre a importância da mediação pedagógica e para que os estudantes assumam posturas mais ativas e aprendam a trabalhar colaborativamente com seus pares, pois "[...] não se comporta [mais] um ensino nas universidades que se caracterize por uma prática pedagógica conservadora, repetitiva e acrítica" (BEHRENS, 2013, p. 75). 


\section{REFERÊNCIAS}

AZEVEDO, Aline Baltazar de; MAIA, Lucas Camelo; SALOMÃO, Ítalo Linhares; CARDOSO, Rafaela Ponte Lisboa; PONTE, Elaine Cristina Rodrigues. Avaliação do desempenho acadêmico na introdução do ensino híbrido em uma disciplina do ciclo profissional de engenharia civil. In: CONGRESSO DE EDUCAÇÃO EM ENGENHARIA, 47.; SIMPÓSIO INTERNACIONAL DE EDUCAÇÃO EM ENGENHARIA DA ABENGE, 2., 17 a 20 de setembro de 2019, Fortaleza. Anais [...]. Fortaleza, CE: Associação Brasileira de Engenharia, 2019. on-line.

BEHRENS, Marilda Aparecida. Projetos de aprendizagem colaborativa num paradigma emergente. In: MORAN, José Manuel; MASETTO, Marcos T.; BEHRENS, Maria Aparecida. Novas tecnologias e mediação pedagógica. Campinas, SP: Papirus, 2013.

BRASIL. Ministério da Educação. Portaria MEC no 343, de 17 de março de 2020. Brasília, DF, 2020.

BRASIL. Ministério da Educação. Referenciais nacionais dos cursos de engenharia. Brasília, DF, 2007.

CETIC. Centro Regional de Estudos para o Desenvolvimento da Sociedade da Informação. Pesquisa sobre o uso das tecnologias de informação e comunicação nos domicílios brasileiros: TIC domicílios 2018. São Paulo: Comitê Gestor da Internet no Brasil, 2019. Disponível em: https://www.cetic.br/media/docs/publicacoes/2/12225320191028tic_dom_2018_livro_eletronico.pdf. Acesso em: 24 jul. 2020.

COLL, César; MONEREO, Carlos. Educação e aprendizagem no século XXI: novas ferramentas, novos cenários, novas finalidades. In: COLL, C; MONEREO, C. (Org.). Psicologia da educação virtual. Porto Alegre, Artmed, 2010.

CRUZ, Dulce Maria. Mediação pedagógica. In: MILL, Daniel (Org.). Dicionário crítico de educação e tecnologia e educação a distância. Campinas, SP: Papirus, 2018.

GARRISON, D. R.; ARBAUGH, J. B. Researching the Community of Inquiry framework: Review, issues and future directions. The internet and higher education, v. 10, n. 3, p. 157 $172,2007$.

GIL, Antonio Carlos. Métodos e técnicas de pesquisa social. São Paulo: Atlas, 2008.

MARTINS, Ronei Ximenes. A COVID-19 e o fim da educação a distância: um ensaio. EmRede: revista de educação a distância, v.7., n.1, p. 242-256, 2020. Disponível em: https://www.aunirede.org.br/revista/index.php/emrede/article/view/620. Acesso em: 30 jul. 2020 .

MATTA, Cláudia Eliane da; CLEMENTINO, Adriana; MONTEVECHI, José Arnaldo Barra. Design instrucional da disciplina de pesquisa operacional baseado no modelo colaborativo. In: CONGRESSO DE EDUCAÇÃO EM ENGENHARIA, 41., 23 a 26 de setembro de 2013, Gramado, RS. Anais [...]. Gramado, RS: Associação Brasileira de Engenharia, 2013. 
MATTA, Cláudia Eliane da; OLIVEIRA, Jane Raquel S. de; FERRAZ, Denise Pereira de Alcantara; LIMA, Rodrigo Silva. Análise da aplicação de uma disciplina de "Cálculo I" a distância para estudantes de cursos de graduação presencial. In: CONGRESSO BRASILEIRO DE ENSINO SUPERIOR A DISTÂNCIA, 12. 2015. Salvador, BA. Anais [...]. Salvador, BA, Unirede, 2015.

MATTA, Cláudia Eliane da; FURLANI, Juliana Maria Sampaio; MORAIS, Jéssica Santana de. Aprendizagem profissional do professor para competências digitais. In: CONFERÊNCIA IBÉRICA DE INOVAÇÃO NA EDUCAÇÃ̃ COM TIC: ieTIC 2020, 6., 2020, Ponta Delgada. Anais [...]. Ponta Delgada (Açores): Instituto Politécnico de Bragança: 2020. on-line.

MORAN, José Manuel. O ensino e a aprendizagem inovadores com o apoio de tecnologias. In: MORAN, José Manuel; MASETTO, Marcos T.; BEHRENS, Maria Aparecida. Novas tecnologias e mediação pedagógica. Campinas, SP: Papirus, 2013.

MASETTO, Marcos T. O ensino e a aprendizagem inovadores com o apoio de tecnologias. In: MORAN, José Manuel; MASETTO, Marcos T.; BEHRENS, Maria Aparecida. Novas tecnologias e mediação pedagógica. Campinas, SP: Papirus, 2013.

RODRIGUES, Alessandra. Ensino remoto na Educação Superior: desafios e conquistas em tempos de pandemia. SBC Horizontes, jun. 2020a. Disponível em: http://horizontes.sbc.org.br/index.php/2020/06/17/ensino-remoto-na-educacao-superior. Acesso em: 25 jul. 2020.

RODRIGUES, Alessandra. Narrativas digitais e experiência: exploração de conceitos e implicações para a educação em uma perspectiva humanista. E-Curriculum, v. 18, n. 2, p. 692714, 2020b. DOI: 10.23925/1809-3876.2020v18i2p692-714. Acesso em: 30 jul. 2020.

ROCKINSON-SZAPKIW, Amanda J.; BAKER, Jason D.; NEUKRUG, Edward; HANES, John. The efficacy of computer mediated communication technologies to augment and support effective online helping profession education. Journal of technology in human services, v. 28, p. 161-177, 2010. DOI: 10.1080/15228835.2010.508363. Acesso em: 30 jul. 2020.

SCHNEIDEIR, Daisy; SILVA, Ketia Kellen Araújo de; BEHAR, Patrícia Alejandra. Competências dos atores na educação a distância: professor, tutor e aluno. In: BEHAR, Patrícia Alejandra (Org.). Competências em educação a distância. Porto Alegre: Penso, 2013. p.152173. 


\title{
PERCEPTIONS OF STUDENTS AND TEACHERS ON THE EXCEPTIONAL TREATMENT REGIME FOR ENGINEERING COURSES
}

\begin{abstract}
During the pandemic period of the new coronavirus, face-to-face classes were replaced by classes in digital media for students at all levels of education. For the resumption of classes, the University referred to in this article developed an exceptional treatment regime, not one of which students received from each teacher a weekly study guide with different teaching strategies, which may or may not include synchronous virtual meetings. After the first weeks, an online questionnaire for students and documents on the implemented regime was prepared. In this article, an analysis of part of the data from a descriptive and exploratory research is presented, with an outline of the perceptions of students and professors of undergraduate courses. It aims to research the positive and negative points pointed out by students about technology-mediated education in the context of emergency remote education and how documents used as digital information and communication technologies to do pedagogical mediation. It was identified that 50.9\% of the students considered it a positive point to continue their studies remotely and that the greatest difficulty faced by them was the excess of weekly tasks. The digital resources most made available by the documents were documents in PDF format and slide shows. He considers that study is relevant for analyzing how perceptions and educational practices with digital technologies during a pandemic, in a Brazilian context and that can contribute to future discussions on digital skills for documents in engineering courses.
\end{abstract}

Keywords: Pedagogical mediation. Digital information and communication technologies. Engineering. Pandemic. 